\title{
Eficácia de um protocolo fisioterapêutico para equilíbrio em idosos institucionalizados
}

\section{Effectiveness of a physiotherapeutic protocol for equilibrium with institutionalized elderly people}

\author{
Mariane Negrão Serra dos Santos Lopes ${ }^{1}$; Cintia Gomes Passerini ${ }^{1}$; Cristiane de \\ Fátima Travensolo ${ }^{2}$
}

\begin{abstract}
Resumo
O equilíbrio consiste em manter o centro de gravidade dentro de uma base de suporte que proporcione maior estabilidade nos segmentos corporais durante situações estáticas e dinâmicas. A manutenção do equilíbrio do corpo no espaço é um fenômeno complexo que depende da integração de várias estruturas como: sistema motor, sensibilidade proprioceptiva, aparelho vestibular, visão, cerebelo dentre outros. A queda é uma das principais conseqüências da falta de equilíbrio no idoso, e a fisioterapia e a atividade física constituem ferramentas importantes para prevenir e/ou minimizar esses déficits. $\mathrm{O}$ objetivo deste estudo foi verificar a eficácia de um programa fisioterapêutico para equilíbrio em idosos institucionalizados. Trata-se de um estudo prospectivo de coorte, do qual participaram sete idosos institucionalizados. Foram incluídos os idosos que deambulavam sem uso de órtese e obedeciam aos comandos e os compreendiam. Foi aplicada uma avaliação inicial dos sistemas respiratório, neurológico e ortopédico e a Escala de Equilíbrio e Mobilidade de Tinetti. O protocolo de tratamento consistiu de 16 terapias, e em cada sessão foi realizado aquecimento, alongamento, fortalecimento, treino de equilíbrio, treino de marcha e relaxamento. Estas foram realizadas duas vezes por semana com duração de 50 minutos cada, em um total de dois meses. Os dados foram estatisticamente avaliados por meio do teste de Wilcoxon com significância de $\mathrm{p}=0,03$, os resultados obtidos mostraram melhora do equilíbrio no grupo estudado. Será sugerido que o protocolo utilizado seja implantado na instituição onde foi realizado, podendo minimizar os riscos inerentes ao déficit de equilíbrio nos idosos.
\end{abstract}

Palavras-Chave: Idosos. Equilíbrio. Instituição.

\begin{abstract}
Equilibrium consists of keeping the gravity center within a support basis which provides more stability in the body segments during static and dynamic situations. The maintenance of body equilibrium in space is a complex phenomenon that depends on several structures, such as: motor system, proprioceptive sensitivity, vestibular system, visual system, cerebellum, among others. The fall is one of the main consequences of lack of equilibrium in the elderly. It is known that the physical therapy and the physical activity are important tools to prevent and/or reduce these deficits. The objective of this work was to verify the effectiveness of a physiotherapeutic program for institutionalized elderly people. Materials and methods: This is a prospective study of cohort, with seven institutionalized elderly people. The elderly who walked without orthosis, were obedient and understood verbal commands, were included. An initial evaluation of the respiratory, neurological and orthopedic systems was used, together with Tinetti's Equilibrium and Mobility Scale. The treatment protocol consisted of 16 therapies. In each session, the warm up, stretching, strengthening, equilibrium training, walk training and relaxation were
\end{abstract}

1 Graduanda em Fisioterapia pelo Centro Universitário Filadélfia (UNIFIL), Londrina-PR. E-mail: marocapinhal@hotmail.com; cgpasserini@hotmail.com

2 Mestre em Gerontologia pela Pontifícia Universidade Católica de São Paulo (PUC-SP); Docente do Centro Universitário Filadélfia (UNIFIL), Londrina-PR; Fisioterapeuta - Universidade Estadual de Londrina (UEL); Especialista em Fisioterapia Cardiorrespiratória -(InCor, FMUSP). E-mail: cristravensolo@hotmail.com 
performed twice a week, with a 50-minute length each, for two months. The data were statistically evaluated through Wilcoxon test with a significance of $p=0.03$. Results showed an improvement in the equilibrium of the studied group. It will be suggested that the protocol used be implemented in the institution where it was conducted, what could reduce the risks inherent in the equilibrium deficit in the elderly.

Key-words: The elderly. Equilibrium. Institution.

\section{Introdução}

O aumento acelerado da população de idosos é um fenômeno mundial observado desde o final do século XIX. Nos países desenvolvidos, o envelhecimento populacional aconteceu gradualmente durante os dois últimos séculos e foi acompanhado pelo crescimento econômico que propiciou a melhoria da qualidade de vida, do saneamento básico, da alimentação, da moradia e uma adequação dos setores de saúde geriátrica e de previdência social (REBELATTO; CASTRO; CHAN, 2007).

No Brasil, a população também passou por uma transição demográfica importante na segunda metade do século XX, com um aumento de 70\% da população de idosos entre os anos de 1950 e 2000, fato que determinou sobrecarga no setor previdenciário, aumento da demanda aos serviços sociais, de saúde e de assistência sanitária (REBELATTO; CASTRO; CHAN, 2007).

As projeções estatísticas apontam que no ano de 2025 o Brasil terá a $6^{\text {a }}$ maior população de idosos do mundo (BITTAR et al., 2007), tornando-se necessário que as políticas públicas existentes sejam aprimoradas e novas políticas sejam elaboradas para implementar o cuidado aos idosos.

O fenômeno de envelhecimento populacional traz consigo uma mudança no perfil de doenças da população, que passa das doenças infectocontagiosas, mais prevalentes nas crianças, para as doenças crônico-degenerativas como as demências e as doenças do sistema cardiovascular, próprias das idades mais avançadas.

O envelhecimento provoca diversas alterações fisiológicas nos órgãos e sistemas. Segundo Faria et al. (2003), essas modificações podem gerar déficits de equilíbrio e alterações da marcha que predispõem o idoso a quedas e limitações funcionais.

Como conseqüências das quedas podem ocorrer fraturas, escoriações e imobilidade, levando à diminuição das atividades de vida diária (AVD's), com prejuízo na qualidade de vida do idoso.

Segundo Faria et al. (2003), os idosos institucionalizados têm maior probabilidade de sofrer quedas do que os idosos não institucionalizados, pois esses possuem menores níveis de força muscular, equilíbrio, flexibilidade e resistência física.

A incapacidade de realizar estas atividades pode ter correlação com o índice de quedas, pois os idosos institucionalizados são mais frágeis, fato que os torna mais propensos a sofrer quedas, por isso eles necessitam de maior atenção e vigilância do que os idosos não institucionalizados (REBELATTO; CASTRO; CHAN, 2007).

Um dos maiores problemas da internação dos idosos nas instituições asilares é a ausência de um programa que estimule a atividade física e cognitiva dessa população. As instituições para idosos de caráter filantrópico são geralmente mantidas por associações religiosas, que normalmente não oferecem atendimento médico nem fisioterápico, a não ser o controle de medicamentos.

A literatura relata que idosos frágeis e institucionalizados podem melhorar os níveis de deficiência, bem como o estado funcional, mediante um programa de exercícios bem idealizados (FARIA et al., 2003). 
Sabe-se que na população geriátrica o déficit de equilíbrio é conseqüência do processo normal do envelhecimento e freqüentemente associase a patologias, cujo resultado são quedas, fraturas, hospitalização e até mesmo a morte. Com base nesses dados, este estudo teve como objetivo geral verificar a eficácia de um protocolo fisioterapêutico para equilíbrio em idosos institucionalizados.

Os objetivos específicos foram: descrever as alterações de equilíbrio, bem como as doenças encontradas nos idosos estudados, testar a eficácia de um protocolo fisioterapêutico de equilíbrio, apresentar os resultados obtidos para a instituição onde ele foi realizado e sugerir a implantação do protocolo de tratamento utilizado.

\section{Material e método}

TIPO DE ESTUDO: Estudo prospectivo de coorte.

CASUÍSTICA: A população selecionada foi composta por um total de sete idosos institucionalizados em uma Instituição de Longa Permanência para Idosos (ILPI) em Londrina PR. A amostragem foi por conveniência.

CRITÉRIO DE INCLUSÃO E EXCLUSÃO: Foram incluídos no estudo todos os idosos que aceitaram participar de livre e espontânea vontade, que deambulavam sem auxílio de órtese e compreendiam os comandos verbais. Para a seleção dos idosos, foi aplicada uma terapia inicial que constou de exercícios metabólicos, alongamentos gerais, um exercício específico para equilíbrio e um exercício específico para marcha. O numero total de idosos participantes desta fase foi 10. Desse grupo, três idosos foram excluídos, pois um participante possuía distúrbio auditivo, outro, distúrbio visual e o terceiro participante não conseguiu terminar a terapia, pois trocava a noite pelo dia e permanecia com sono durante a terapia. Foram excluídos do estudo aqueles idosos que possuíam amputações, que deambulavam com o auxilio de órtese e ou eram acamados.

COLETA DE DADOS: Inicialmente e ao final do tratamento, foi aplicada uma avaliação fisioterapêutica individual e a Escala de Equilíbrio e Mobilidade de Tinetti, respectivamente no primeiro e terceiro bimestres de 2008 . Os resultados foram analisados no início da intervenção e ao final, comparando-se os dois momentos. Foram realizados dois meses de intervenção, perfazendo um total de 16 terapias, realizadas duas vezes por semana com duração de 50 minutos cada.

\section{Protocolo de intervenção}

Antes e ao final de cada terapia, eram verificados os dados vitais, como freqüência cardíaca, freqüência respiratória e pressão arterial.

\section{Terapias de 1 a 4}

Aquecimento: exercícios metabólicos de MMSS e MMII (5 minutos).

Alongamentos gerais de MMSS e MMII (1x20).

Fortalecimento isométrico de bíceps, tríceps e quadríceps $(2 \times 10)$.

Equilíbrio: andar cinco passos dar uma volta no banco e voltar cinco passos (1 série).

Marcha: andar no pátio (uma série).

Relaxamento: andar no pátio e relaxar MMSS, MMII, pescoço e ombros (10 minutos).

Terapias de 5 a 8

Aquecimento: exercícios metabólicos de MMSS e MMII (5 minutos).

Alongamentos gerais de MMSS e MMII (1x20).

Fortalecimento isotônico de bíceps, tríceps e quadríceps com halter de 500g (2x10). 
Equilíbrio: andar cinco passos dar uma volta no banco pular três bastões e voltar cinco passos (uma série).

Marcha: andar em linha reta (uma série).

Relaxamento: andar no pátio e relaxar MMSS, MMII, pescoço e ombros (10 minutos).

\section{Terapias de 9 a 12}

Aquecimento: exercícios metabólicos de MMSS associado com caminhada (5 minutos).

Alongamentos gerais de MMSS e MMII (1x20).

Fortalecimento isotônico de bíceps, tríceps e quadríceps com halter de 500g (3x10).

Equilíbrio: andar cinco passos, dar uma volta no banco, pular três bastões e um step e voltar cinco passos (uma série).

Marcha: andar em linha reta e andar de lado (uma série)

Relaxamento: andar no pátio e relaxar MMSS, MMII, pescoço e ombros (10 minutos).

\section{Terapias de 13 a 16}

Aquecimento: exercícios metabólicos de MMSS associado com caminhada (5 minutos).

Alongamentos gerais de MMSS e MMII (1x20).

Fortalecimento isotônico de bíceps, tríceps e quadríceps com halter de 500g (3x10).

Equilíbrio: andar cinco passos, dar uma volta no banco, pular três bastões, pular um step e passar por 3 cones e voltar cinco passos (uma série).

Marcha: andar em linha reta, andar de lado e andar com circuitos (umasérie).

Relaxamento: andar no pátio e relaxar MMSS, MMII, pescoço e ombros (10 minutos).
Análise de Dados: As variáveis da Escala de Equilíbrio e Mobilidade de Tinetti são qualitativas ordinais (normal, adaptável, anormal). Elas foram analisadas estatisticamente, com o uso do teste de Wilcoxon.

Aspectos Éticos: Este trabalho foi apresentado e aprovado pelo Comitê de Ética em Pesquisa do Centro Universitário Filadélfia (Unifil), Oficio $n^{0} 96 / 080$, e autorizado pela responsável da Instituição de Longa Permanência. Todos os idosos foram esclarecidos acerca do objetivo do trabalho e somente participaram aqueles que assinaram o Termo de Consentimento Livre Esclarecido.

\section{Resultados}

Os resultados são apresentados segundo média e desvio padrão. A média de idade dos idosos participantes é 75,5 $\pm 7,9$ anos, e todos pertencem ao gênero masculino totalizando sete indivíduos. Foi excluído um indivíduo que reencontrou a família e deixou a instituição.

Entre os dados da avaliação fisioterapêutica constam que quatro destes residem há dois anos na Instituição e os dois restantes moram nela a menos de um ano. No que diz respeito à dor, somente três indivíduos apresentaram dor no dia da avaliação inicial, avaliada pela escala analógica da dor, e foram encontrados sintomas de cefaléia, dor em região dorsal e em membros inferiores.

Dentre os seis participantes, três idosos tinham hipertensão arterial controlada com uso de medicação diária, três possuíam outras doenças associadas, sendo dois Doença de Alzheimer na fase inicial e um distúrbio de comportamento. Foi encontrado déficit visual em dois idosos, mas estes utilizavam órtese adequada. Esses dados podem ser verificados na tabela 1 : 
Tabela 1. Características dos indivíduos de instituições de longa permanência para idosos em Londrina, PR.

\begin{tabular}{lllllll}
\hline Paciente & $\begin{array}{c}\text { Idade } \\
\text { (anos) }\end{array}$ & $\begin{array}{c}\text { Tempo de } \\
\text { instituição } \\
\text { (meses) }\end{array}$ & $\begin{array}{c}\text { Presença } \\
\text { de dor }\end{array}$ & $\begin{array}{c}\text { Hipertensão } \\
\text { arterial }\end{array}$ & $\begin{array}{c}\text { Outras } \\
\text { doenças } \\
\text { associadas }\end{array}$ & Órtese \\
\hline 1 & 71 & 24 & Sim & Sim & Alzheimer & Não \\
2 & 84 & 11 & Não & Não & Não & Não \\
3 & 77 & 24 & Não & Não & Alzheimer & Óculos \\
4 & 78 & 8 & Não & Sim & Não & Não \\
5 & 62 & 24 & Sim & Sim & Não & Óculos \\
6 & 81 & 24 & Sim & Não & Distúrbio & Não \\
& & & & de & comportamento & \\
\hline
\end{tabular}

Os resultados que seguem são apresentados segundo a média, desvio padrão e mediana, na avaliação por meio da Escala de Equilíbrio e Mobilidade de Tinetti. $\mathrm{Na}$ avaliação inicial, os pacientes do grupo obtiveram a média de $17,8 \pm 1,9$ e a mediana de 17,5 pontos, com máximo de 21 e mínimo de 16 pontos. $\mathrm{Na}$ avaliação final, obteve-se a média de 24,1 $\pm 3,1$ e mediana de 25 , com o valor máximo de 27 e mínimo de 18 pontos.

A escala de Equilíbrio e mobilidade de Tinetti é separada em dois quesitos: equilíbrio e marcha, e tem como escore total no quesito equilíbrio 16 pontos. Quanto menor a pontuação, maior o déficit de equilíbrio.

$\mathrm{Na}$ avaliação inicial, os participantes obtiveram média neste quesito de $10,5 \pm 1,6$ pontos e mediana de 10,5, com o valor máximo de 12 e mínimo de 9 pontos. Na reavaliação, a média foi de $13,8 \pm 1,9$ pontos e mediana de 14, 5, com valor máximo de 15 e mínimo de 10 pontos.

No quesito marcha da Escala de equilíbrio e mobilidade de Tinetti, a pontuação máxima é de 12 pontos e, quanto menor a pontuação, maior o déficit na marcha.

$\mathrm{Na}$ avaliação inicial, o grupo obteve a média de 7,3 $\pm 1,8$ pontos e mediana de 7,5 , com o valor máximo de 9 e mínimo de 4 pontos. Na reavaliação obteve-se a média de $10,3 \pm 1,3$ pontos e mediana de 10,5 , com valor máximo de 12 e mínimo de 8 pontos.

Estes dados podem ser verificados a seguir na tabela 2.

Tabela 2. Escala de equilíbrio e mobilidade de Tinetti de idosos de instiuições de longa permanência em Londrina,

\begin{tabular}{lll}
\hline Escala & Media $\pm \mathbf{d p}$ & Mediana \\
\hline Tinetti inicial & $17,8 \pm 1,9$ & 17,5 \\
Tinetti Final & $24,1 \pm 3,1$ & 25 \\
Tinetti equilíbrio inicial & $10,5 \pm 1,6$ & 10,5 \\
Tinetti equilíbrio final & $13,8 \pm 1,9$ & 14,5 \\
Tinetti marcha inicial & $7,3 \pm 1,8$ & 7,5 \\
Tinetti marcha final & $10,3 \pm 1,3$ & 10,5 \\
\hline
\end{tabular}


Para a análise estatística dos dois momentos da avaliação (inicial e final) foi utilizado o teste de Wilcoxon, por meio do qual analisou-se a Escala de Equilíbrio e Mobilidade de Tinetti total e os quesitos equilíbrio e marcha, separadamente.

Nos resultados da Escala de Equilíbrio e
Mobilidade de Tinetti total e Tinetti marcha, os valores obtidos apresentaram significância com $p=0,03$. No quesito Tinetti equilíbrio os resultados também foram estatisticamente significantes, com $\mathrm{p}=0,05$.

Os resultados estão dispostos a seguir na Tabela 3.

Tabela 3. Teste de Wilcoxon sobre as escalas específicas de Tinetti de idosos de instituições de longa permanência, londrina, PR.

\begin{tabular}{lll}
\hline Escala & Teste & $\mathbf{p}$ \\
\hline Tinetti Total & Wilcoxon & 0,03 \\
Tinetti equilíbrio & Wilcoxon & 0,05 \\
Tinetti marcha & Wilcoxon & 0,03 \\
\hline
\end{tabular}

\section{Discussão}

Estima-se em 85\% a prevalência de déficit de equilíbrio na população acima de 65 anos. Cerca de $30 \%$ dos idosos que vivem na comunidade caem ao menos uma vez ao ano, mas entre os idosos institucionalizados essa prevalência sobe para cerca de $60 \%$ a $75 \%$. Estudos têm se dirigido para melhora do equilíbrio em idosos, principalmente para os institucionalizados, pois a institucionalização contribui para a perda da autonomia nesta população, tornando-a mais frágil (BITTAR et al., 2007).

A independência funcional requer recrutamento motor, equilíbrio, resistência cardiovascular e também motivação. Costuma-se afirmar que a deterioração dessas capacidades é inevitável com o envelhecimento, mas está claro que muito dessa deterioração pode ser atribuída à inatividade. Isso significa que a implantação de um programa de exercícios terapêuticos, mesmo em idades extremas, é capaz de minimizar ou mesmo evitar o declínio funcional acentuado, amenizando os efeitos das doenças ou mesmo prevenindo-as (FARIA et al., 2003).

Este estudo abrangeu somente o gênero masculino, embora estudos que verificam a eficácia de equilíbrio em idosos geralmente se voltam para o gênero feminino, pois as mulheres vivem mais e, conseqüentemente, são mais institucionalizadas. Porém na instituição em que foi realizado o estudo as mulheres apresentavam grau importante de déficit cognitivo e maior comprometimento motor quando comparadas aos homens, e por serem na sua maioria acamadas não tiveram participação neste estudo. A idade média foi 75,5 $\pm 7,9$ anos.

Ao comparar o presente estudo com os demais encontrados na literatura, pode-se observar a semelhança com a idade média encontrada entre eles. Bittar et al. (2007) analisaram 55 pacientes com queixa de desequilíbrio corporal, caracterizando o perfil desta população de idosos quanto à idade e gênero. Dos 55 pacientes avaliados, 45 eram do gênero feminino e 10 do masculino, a média de idade dos pacientes foi de 72,3 anos, desvio padrão de $\pm 6,46$ anos e mediana de 71 anos. No estudo de Ribeiro e Pereira (2005) a idade média foi de 64,8 $\pm 2,95$ anos e a maior prevalência foi do gênero feminino.

Os autores citam que quanto maior a idade média dos participantes maior será o risco de quedas, pois os idosos com idade avançada possuem maior desequilíbrio corporal e estão mais sujeitos a este risco. Neste estudo, nas 16 terapias realizadas, nenhum idoso sofreu quedas, vertigens, fadiga ou 
desequilíbrio corporal.

Quanto ao tempo de intervenção e a freqüência das terapias, Zambaldi et al. (2007) realizaram o treinamento em atividades em grupo, duas vezes por semana com uma hora de duração cada, por um período de oito semanas. Já no estudo de Ribeiro e Pereira (2005), as terapias tiveram duração de nove semanas, com freqüência de duas vezes por semana e duração de uma hora cada. No estudo de Bechara e Santos (2008), os autores realizaram seu treinamento duas vezes por semana, por um período de uma hora cada terapia, durante seis semanas, totalizando 12 terapias.

No presente estudo as atividades foram realizadas em grupo durante oito semanas com duas sessões semanais, totalizando 16 terapias, mostrando semelhança com a literatura pesquisada.

Estudos que buscam avaliar o equilíbrio em idosos demonstram que as escalas mais confiáveis para avaliação deste quesito são as escalas de Berg, 1992 (MIYAMOTO, et al., 2004) e Escala de Equilíbrio e Mobilidade de Tinetti, 1986 (FREITAS et al., 2002).

Nos trabalhos de Ribeiro e Pereira (2005), Deleski, Silva e Alvarenga (2007) e Zambaldi et al. (2007), foram utilizadas as escala de Berg para estudar o equilíbrio da população idosa pesquisada. Já no estudo de Cattuzzo et al. (2005), foi utilizada a escala de equilíbrio e mobilidade de Tinetti.

Por meio desses estudos, nota-se que tanto a Escala de Berg quanto a Escala de Equilíbrio e Mobilidade de Tinetti podem ser utilizadas para avaliação do equilíbrio, e que em ambas quanto menor a pontuação maior será o déficit de equilíbrio.

No presente estudo foi utilizada a Escala de Equilíbrio e Mobilidade de Tinetti, que segundo Ribeiro e Pereira (2005), mostra-se confiável na avaliação funcional e revela objetividade de testereteste (RIBEIRO; PEREIRA, 2005).

Zambaldi et al. (2007) avaliaram um grupo de sete idosas com idade média de 79,1 $\pm 2,91$ anos, todas portadoras de alterações de equilíbrio. $\mathrm{O}$ protocolo de tratamento consistiu de atividades realizadas em grupo a partir de exercícios isolados e circuitos fechados, com os treinamentos realizados duas vezes por semana, com uma hora de duração, por um período de oito semanas totalizando dezesseis terapias. $\mathrm{O}$ estudo mostrou-se eficaz na melhoria de equilíbrio dessas mulheres.

Já no estudo de Bechara e Santos (2008), os autores avaliaram um grupo de 10 idosos, dos quais oito pertenciam ao gênero masculino e dois pertenciam ao feminino, sendo estes não institucionalizados, com idade média de $66,1 \pm 6,12$ anos. As terapias basearam-se no conceito Bobath, evidenciaram melhora estatisticamente significante a favor do protocolo de equilíbrio para idosos.

Neste estudo, a idade média dos participantes foi $75,5 \pm 7,9$ anos, todos pertencentes do gênero masculino e com desequilíbrio corporal. Eles tinham como protocolo de tratamento: aquecimento articular, alongamentos gerais de membros superiores e inferiores, fortalecimento de grupos musculares específicos, como bíceps, tríceps e quadríceps, exercícios para equilíbrio em circuito e exercícios para marcha.

Nas terapias, foram apresentados exercícios em solo, com enfoque maior nos exercícios de equilíbrio, associados aos exercícios de membros superiores e inferiores. A terapia em grupo, realizada com os idosos no presente estudo, estimulou a interação e a melhora do convívio entre eles, que não se conheciam, embora residissem na mesma instituição.

Todas as terapias foram realizadas em grupo, duas vezes por semana, com duração de cinqüenta minutos cada, por um período de oito semanas totalizando dezesseis terapias. Houve melhora significativa do equilíbrio na população estudada.

Como o objetivo primordial de uma intervenção fisioterapêutica em idosos é restabelecer ou preservar a função motora, a intervenção realizada com exercícios de fortalecimento muscular é uma 
valiosa alternativa, que, além de eficaz, tem uma excelente relação custo-benefício. Os exercícios podem ser executados de forma independente pelo idoso, entretanto é necessário monitoramento adequado pelo profissional de saúde em todas as fases do treinamento, para evitar lesões e otimizar os resultados (FARIA et al., 2003).

Outro enfoque nas terapias foi a marcha, pois a eficiência desta se deteriora com o avançar da idade devido a certas mudanças como o encurtamento muscular e a diminuição da altura do passo, o alargamento da base de sustentação, a diminuição da velocidade da marcha, além do aumento da fase de apoio e do tempo de duplo suporte (apoio bipodal), de modo que há maior gasto energético para realizá-la. Segundo Faria et al. (2003), essas alterações podem desencadear diminuição em outras atividades desempenhadas, motivo pelo qual foi essencial a inclusão de exercícios específicos para marcha no protocolo implantado.

Este estudo mostrou concordância com os dados encontrados na literatura no que se refere ao tempo de intervenção, freqüência das terapias, atividades propostas no protocolo e idade média da população.

Em relação ao gênero, predominou o feminino na bibliografia pesquisada, pois esta população vive mais e conseqüentemente é mais institucionalizada, porém na população do estudo predominou a masculina porque as mulheres eram em sua maioria acometidas por déficit motor, fatos que as impediu de participarem das terapias.

O protocolo de equilíbrio obteve resultados significantes para a população estudada e pode ser utilizado para tratamento fisioterapêutico de idosos que possuem desequilíbrio corporal, porém há necessidade de novos estudos sobre equilíbrio na população idosa.

Será sugerida a implantação do protocolo de tratamento na instituição onde foi realizado este estudo, e ele poderá fazer parte do estágio curricular supervisionado em campo obrigatório para os alunos do $4^{\circ}$ ano do Curso de Fisioterapia do Centro
Universitário Filadélfia (Unifil).

\section{Considerações finais}

O protocolo implantando foi capaz de melhorar o equilíbrio nos idosos institucionalizados participantes, e essa melhora é estatisticamente significante. Há necessidade de estudos adicionais acerca do equilíbrio em idosos, com um número maior de participantes, objetivando reduzir o erro tipo I. Espera-se, assim, que este estudo possa contribuir para estudos futuros sobre o equilíbrio na população idosa.

\section{Referências}

BECHARA, F. T.; SANTOS, S. S. M. Efetividade de um programa fisioterapêutico para treino de equilíbrio em idosos. Revista Saúde e Pesquisa, Londrina, v. 1, n. 1, p. 5-20, 2008.

BITTAR, R. S. M.; SIMOCELI, L.; PEDALINI, M. E. B.; BOTTINO, M. A. Repercussão das medidas de correção das comorbidades no resultado da reabilitação vestibular de idosos. Revista Brasileira de Otorrinolaringologia, São Paulo, v. 73, n. 3, p. 295-298, 2007.

CATTUZZO, M. T.; WALTER, C.; BASSO, L.; CASTRO,W. V.; MIRANDA, T. A. Analise do desempenho de idosos em tarefas de equilíbrio. Revista Brasileira de Atividade Física e Saúde, Londrina, v. 9, n. 2, p. 12-22, 2005.

DELESKI, E. V.; SILVA, F. C.; ALVARENGA, L. F. Comparação da qualidade de vida e equilibrio funcional entre idosos praticantes de exercícios físicos e sedentários. In: CONGRESSO SUL-BRASILEIRO DE ORTOPEDIA E TRAUMATOLOGIA - SULBRA, 15. Gramado, 2007. Anais... Gramado, 2007.

FARIA, J. C.; MACHALA, C. C.; DIAS, R. C.; DIAS, J. M. $D$ Importância do treinamento de força na reabilitação da função muscular, equilíbrio e mobilidade de idosos. Acta Fisiátrica, São Paulo, v. 10, n. 3, p. 133-137, 2003.

FREITAS, E. V.; PY, L.; NERI, A. L.; CANÇADO, F. A. X.; GORZONI, M. L.; ROCHA, S. M. Tratado de geriatria e gerontologia. Rio de Janeiro: GuanabaraKoogan, 2002.

MIYAMOTO, S. T.; LOMBARDI JUNIOR, I.; BERG, K. O.; RAMOS, L. R.; NATOUR, J. Brazilian version of the Berg balance scale. Brazilian Journal of Medical and Biological Research, Ribeirão Preto, v. 37, n. 9, p. 1411-1421, 2004. 
REBELATTO, J. R.; CASTRO, A. P.; CHAN, A. Quedas em idosos institucionalizados: características gerais, fatores determinantes e relações com a força de preensão manual. Acta Ortopédica Brasileira, São Paulo, v. 15, n. 3, p. 151-154, 2007.

RIBEIRO, A. S. B.; PEREIRA, J. S. Melhora do equilíbrio e redução da possibilidade de queda em idosas após os exercícios de Cawthorne e Cooksey. Acta Ortopédica Brasileira, São Paulo, v. 15, n. 3, p. 151-154, 2005.

ZAMBALDI, P. A.; COSTA, T. A. B. N.; DINIZ, G. C. L. M.; SCALZO, P. L. Efeito de um treinamento de equilíbrio em um grupo de mulheres idosas da comunidade: estudo piloto de uma abordagem específica, não sistematizada e breve. Acta Fisiátrica, São Paulo, v. 14, n. 1, p. 17-24, 2007.

Recebido em: 15 de julho de 2009

Aceito em: 09 de junho de 2010 